Handbook of Sweeteners 


\title{
Handbook of Sweeteners
}

\author{
Edited by \\ S. MARIE \\ Meat and Livestock Commission \\ Winterhill House \\ Milton Keynes \\ and \\ J.R. PIGGOTT \\ Food Science Laboratories \\ Department of Bioscience and Biotechnology \\ University of Strathclyde
}

Springer Science+Business Media, LLC 
$\begin{array}{llllllllllllllll}16 & 15 & 14 & 13 & 12 & 11 & 10 & 9 & 8 & 7 & 6 & 5 & 4 & 3 & 2 & 1\end{array}$

(C) 1991 Springer Science+Business Media New York Originally published by Blackie and Son Ltd in 1991

First published 1991

All rights reserved.

No part of this publication may be reproduced, stored in a retrieval system, or transmitted, in any form or by any means -

graphic, electronic or mechanical, including photocopying, recording, taping - without the written permission of the Publishers

\section{British Library Cataloguing in Publication Data}

Handbook of sweeteners.

I. Marie, S. II. Piggott, J.R.

664

ISBN 978-1-4757-5382-0

\section{Library of Congress Cataloging-in-Publication Data}

Handbook of sweeteners / edited by S. Marie and J.R. Piggott.

p. $\mathrm{cm}$.

Includes bibliographical references.

ISBN 978-1-4757-5382-0

ISBN 978-1-4757-5380-6 (eBook)

DOI 10.1007/978-1-4757-5380-6

1. Sweeteners. I. Marie. S. (Susan). II. Piggott, J.

R. (John Raymond).

TP421.H36 1991

$664^{\prime} .1-\mathrm{dc} 20$

91-6307

CIP

Phototypesetting by Selectmove Ltd, London 


\section{Preface}

The study of sweetness and sweeteners has recently been an area wellserved by books at all levels, but this volume was planned to fill what we perceived as a gap in the coverage. There appeared to be no book which attempted to combine a study of sweetness with a thorough but concise coverage of all aspects of sweeteners. We set out to include all the important classes of sweeteners, including materials which do not yet have regulatory approval, so that clear comparisons could be made between them and their technological advantages and disadvantages. To achieve our first aim, of sufficient depth of coverage, the accounts within this volume are comprehensive enough to satisfy the requirements of a demanding readership, but cannot be exhaustive in a single volume of moderate proportions. The second aim, of breadth and conciseness, is satisfied by careful selection of the most pertinent material.

For the purposes of this book, a sweetener is assumed to be any substance whose primary effect is to sweeten a food or beverage to be consumed, thus including both the nutritive and non-nutritive varieties, from the ubiquitous sucrose to the lesser known, newer developments in alternative sweeteners.

The volume has its contents structured in a logical manner to enable it to be used in an ordered study of the complete subject area or as a convenient reference source. The book opens with a study of sweetness and its influence on food selection, to set the scene for the following chapters discussing how this demand for sweetness is satisfied, and its implications. The following five chapters discuss the major classes of sweeteners, including their sources and technological applications. The following chapter discusses the marketing of sweeteners and their uses in product development. No study of sweeteners can be complete without a consideration of the health implications of what may constitute a significant proportion of the diet, and this is included in the next three chapters covering dental health, metabolic disorders and body weight. The final chapter considers the legislative framework providing for control of sweeteners as food ingredients. 
It is anticipated that this volume will be read by all those seeking an authoritative review of the whole subject of sweeteners in a single volume. Its appeal should thus be wide, attracting readers such as food scientists and technologists, and developers of food, beverage and pharmaceutical products. Selective reading is also expected by workers concerned with dental health, metabolic disorders such as diabetes mellitus, and weight control, as well as those, such as psychologists interested in sweetness as a perceptual phenomenon, seeking background information.

The reader must judge whether we have succeeded in our aim of producing a complete but concise review of sweetness and sweeteners. If so, it is due to the help of all those concerned: the many contributors who have worked hard to turn our ideas into print; and the publisher's staff, for their encouragement, patience, and skilled work. 


\section{Contributors}

M.S. Billaux Association des praticiens pour l'information en nutrition et diététique (APRID), 64 rue de Miromesnil, F-75008 Paris, France.

D. Birkhed Department of Cariology, Faculty of Odontology, University of Göteborg, Box 33070, S-400 33 Göteborg, Sweden.

D.A. Booth School of Psychology, Food and Nutrition Laboratory, University of Birmingham, Edgbaston, Birmingham B15 2TT, UK.

M.A. Clarke Sugar Processing Research, Inc., 1100 Robert E. Lee Boulevard, New Orleans, Louisiana 70124, USA.

M.T. Conner Department of Psychology, University of Leeds, Leeds LS2 9JT, UK.

G.E. Dubois The NutraSweet Company, 601 E. Kensington Road, Mt. Prospect, Illinois 60056-1300, USA.

C.-G. Emilson Department of Cariology, Faculty of Odontology, University of Göteborg, Box 33070, S-400 33 Göteborg, Sweden.

N. Finer Luton and Dunstable Hospital, Lewsey Road, Luton LU4 0DZ, UK.

B. Flourié INSERM U290, Hôpital Saint-Lazare, Paris, France.

R.C. Gelardi Calorie Control Council, Suite 500-D, 5775 PeachtreeDunwoody Road, Atlanta, Georgia 30342, USA.

C. Jacquemin Agriculture, Nutrition Développement (AND), Paris, France.

S.-H. Kim Department of Chemistry and Lawrence Berkeley Laboratory, University of California, Berkeley, California 94720, USA.

M.G. Lindley Lintech, Reading University Innovation Centre, PO Box 68, Whiteknights, Reading RG6 2BX, UK.

B. Messing Service de gastroentérologie et assistance nutritive, Hôpital Saint-Lazare, Paris, France. 
viii CONTRIBUTORS

L. O'Brien Nabors Calorie Control Council, Suite 500-D, 5775 PeachtreeDunwoody Road, Atlanta, Georgia 30342, USA.

W.M. Nicol Eurobest Associates, Dervaig House, 83a Kidmore Road, Caversham, Reading RG4 7NQ, UK.

D.J. Snodin Eurobest Associates, Westview, Fairfield Road, Goring-onThames, Reading RG8 0EY, UK.

K. Wennerholm Department of Cariology, Faculty of Odontology, University of Göteborg, Box 33070, S-400 33 Göteborg, Sweden. 


\section{Contents}

1 Sweetness and food selection 1

M.T. CONNER

1.1 Introduction 1

1.2 Innate versus learned preference for sweetness 2

1.2.1 Studies with newborn infants

1.2.2 Studies with older infants

1.2.3 The role of dietary experience 4

1.2.4 The mature response to sweetness 5

1.3 The linear food acceptance function 9

$\begin{array}{ll}\text { 1.3.1 The acceptance triangle } & 10\end{array}$

1.3.2 Psychophysical acceptance parameters 13

1.3.3 The aggregation of individual acceptance responses 15

1.3.4 Combined action of several determinants of acceptance 16

$\begin{array}{lll}1.4 & \text { Sweetness and food selection } & 17\end{array}$

$\begin{array}{lll}\text { 1.4.1 The sweet tooth defined } & 17\end{array}$

1.4.2 Partial assessment of the sweet tooth construct 17

$\begin{array}{ll}\text { 1.4.3 Full assessment of the sweet tooth construct } & 18\end{array}$

$\begin{array}{lll}1.5 & \text { Other sensory factors affecting sweetener perception } & 23\end{array}$

1.6 Satiety and sweetness preference $\quad 24$

$\begin{array}{ll}\text { 1.6.1 Sensory-specific satiety } & 25\end{array}$

1.6.2 Effects of intense sweeteners

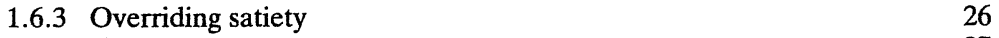

$\begin{array}{lll}1.7 & \text { Conclusions } & 27\end{array}$

$\begin{array}{lr}\text { References } & 28\end{array}$

2 The carbohydrate-sucrose 33

W.M. NICOL

$\begin{array}{lll}2.1 & \text { Source } & 33\end{array}$

2.2 Development and processing 34

2.2.1 Beet processing 35

2.2.2 Cane processing 37

2.2.3 Refining 38

2.3 Properties $\quad 39$

2.3.1 Sensory properties $\quad 40$

2.3.2 Physical properties $\quad 42$

2.4 Health and nutrition $\quad 46$

$\begin{array}{lll}2.4 .1 & \text { Nutrition } & 46\end{array}$

2.4.2 Cariogenic potential $\quad 47$ 
$\begin{array}{lll}2.5 & \text { Strengths and limitations } & 48\end{array}$

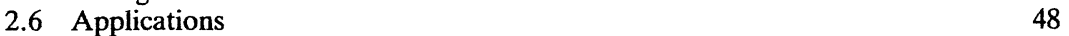

2.6.1 Confectionery 48

$\begin{array}{llr}2.6 .2 & \text { Baking } & 49\end{array}$

2.6.3 Soft drinks $\quad 50$

2.6.4 Preserves $\quad 50$

2.7 Conclusion $\quad 51$

References $\quad 51$

3 Non-sucrose carbohydrates $\quad 52$ M.A. CLARKE

3.1 Introduction $\quad 52$

3.2 Honey $\quad 53$

3.2.1 Composition and properties 53

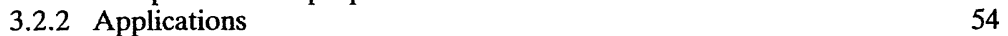

3.3 Maple syrup and sugar $\quad 55$

3.4 Molasses and cane syrups $\quad 55$

3.4.1 Composition and properties $\quad 55$

3.4.2 Applications $\quad 59$

3.4.3 Cane syrup $\quad 59$

$\begin{array}{ll}3.4 .4 & \text { Golden syrup }\end{array}$

$\begin{array}{ll}3.4 .5 & \text { Sorghum syrup }\end{array}$

$\begin{array}{lll}3.4 .6 & \text { Fruit syrups } & 61\end{array}$

3.5 Disaccharides other than sucrose $\quad 62$

$\begin{array}{ll}3.5 .1 & \text { Lactose and lactulose }\end{array}$

$\begin{array}{lll}3.5 .2 & \text { Palatinose } & 62\end{array}$

$\begin{array}{lll}3.5 .3 & \text { Leucrose } & 63\end{array}$

$\begin{array}{ll}3.5 .4 \text { Maltose } & 63\end{array}$

$\begin{array}{lll}3.6 & \text { Oligosaccharides } & 64\end{array}$

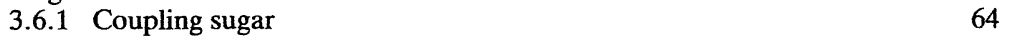

$\begin{array}{ll}3.6 .2 \text { Neosugar } & 64\end{array}$

$\begin{array}{lll}3.7 & \text { Starch-based sweeteners } & 65\end{array}$

$\begin{array}{ll}\text { 3.7.1 Manufacturing process } & 67\end{array}$

$\begin{array}{lll}3.8 & \text { High fructose corn syrups } & 68\end{array}$

$\begin{array}{ll}\text { 3.8.1 Crystalline fructose } & 68\end{array}$

$\begin{array}{ll}3.8 .2 & \text { Dextrose syrups }\end{array}$

3.8.3 Dextrose anhydrous $\quad 69$

3.8.4 High maltose syrups $\quad 69$

3.8.5 Chemical and physical properties $\quad 69$

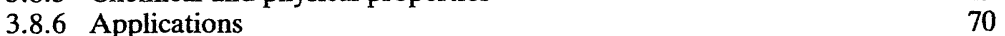

$\begin{array}{ll}\text { Acknowledgements } & 71\end{array}$

$\begin{array}{ll}\text { References } & 71\end{array}$

4 Sugar alcohols $\quad \mathbf{7 2}$

M.S. BILLAUX, B. FLOURIÉ, C. JACQUEMIN and B. MESSING

$\begin{array}{lll}4.1 & \text { Sources, development and processing } & 72\end{array}$

$\begin{array}{lll}\text { 4.1.1 Sorbitol } & 72\end{array}$

4.1.2 Mannitol 73

$\begin{array}{ll}4.1 .3 \text { Xylitol } & 74\end{array}$

4.1.4 Isomalt $\quad 75$

$\begin{array}{ll}\text { 4.1.5 Maltitol } & 75\end{array}$

$\begin{array}{ll}\text { 4.1.6 Lactitol } & 75\end{array}$

$\begin{array}{ll}\text { 4.1.7 Hydrogenated glucose syrups } & 75\end{array}$

$\begin{array}{ll}4.2 & \text { Physical and chemical properties } \\ \end{array}$ 
4.2.1 Molecular weight $\quad 79$

4.2.2 Density $\quad 79$

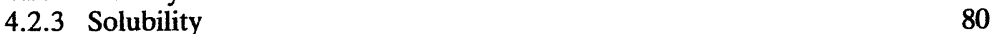

$\begin{array}{lll}4.2 .4 & \text { Viscosity } & 81\end{array}$

4.2.5 Refractive index

4.2.6 Rotatory power $\quad 82$

4.2.7 Hygroscopicity $\quad 82$

4.2.8 Boiling temperature $\quad 82$

4.2.9 Freezing temperature $\quad 84$

$\begin{array}{ll}4.2 .10 \text { Heat of solution } & 84\end{array}$

4.2.11 Reducing properties $\quad 84$

4.2.12 Bacteriological properties $\quad 85$

4.2.13 Sensory properties $\quad 85$

$\begin{array}{ll}\text { 4.2.14 Synergistic effect } & 85\end{array}$

4.3 Applications of polyols $\quad 86$

4.3.1 Biscuit-making and bread-making 86

$\begin{array}{ll}\text { 4.3.2 Non-alcoholic beverages } & 87\end{array}$

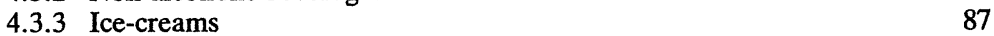

$\begin{array}{lll}\text { 4.3.4 Confectionery } & 88\end{array}$

4.3.5 Jams, jellies, fruit preserves 91

4.3.6 Dairy products

4.3.7 Pharmaceutical and cosmetic applications $\quad 92$

$\begin{array}{lll}4.4 & \text { Legislative factors } & 92\end{array}$

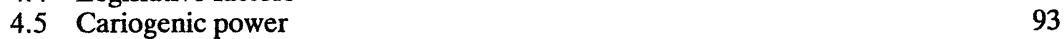

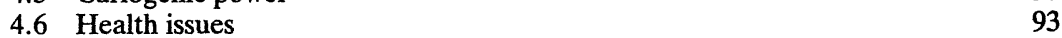

4.6.1 Digestion of polyols in the human digestive tract 93

4.6.2 Factors controlling the degree of digestion-absorption,
clinical tolerance and energy value of polyols

4.6.3 The main polyols $\quad 96$

4.6.4 Sugar alcohols and health 100

$\begin{array}{ll}\text { References } & 100\end{array}$

5 Intense sweeteners $\quad 104$

L. O'BRIEN NABORS and R.C. GELARDI

$\begin{array}{lll}5.1 & \text { Introduction } & 104\end{array}$

$\begin{array}{lll}5.2 & \text { Aspartame } & 105\end{array}$

5.3 Saccharin 105

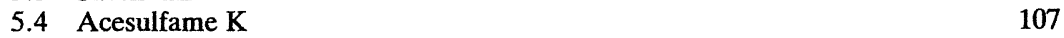

$\begin{array}{lr}5.5 \text { Cyclamate } & 109\end{array}$

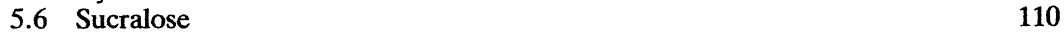

$\begin{array}{ll}5.7 \text { Alitame } & 112\end{array}$

$\begin{array}{lll}5.8 & \text { L-Sugars } & 113\end{array}$

5.9 Sweeteners in their infancy 114

$\begin{array}{ll}\text { References } & 114\end{array}$

6 Natural high potency sweeteners 116

S.-H. KIM and G.E. DUBOIS

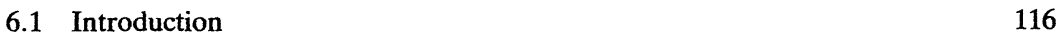

$\begin{array}{lll}6.2 & \text { Protein sweeteners } & 117\end{array}$

6.2.1 Thaumatin and monellin $\quad 118$

6.2.2 Mabinlin, pentadin, curculin and others $\quad 129$

6.3 Peptide sweeteners 131

$\begin{array}{ll}\text { 6.3.1 Aspartame } & 131\end{array}$

$\begin{array}{ll}\text { 6.3.2 Other peptide sweeteners } & 135\end{array}$ 
$\begin{array}{lll}6.4 & \text { Terpenoid sweeteners } & 137\end{array}$

$\begin{array}{lll}\text { 6.4.1 } & \text { Monoterpenoids } & 138 \\ \text { 6.4.2 } & \text { Sesquiterpenoids }\end{array}$

$\begin{array}{ll}6.4 .3 \text { Diterpenoids } & 139\end{array}$

$\begin{array}{ll}\text { 6.4.4 Triterpenoids } & 152\end{array}$

6.5 Polyketide sweeteners 158

6.6 Requirements for commercial viability of non-nutritive sweeteners 170

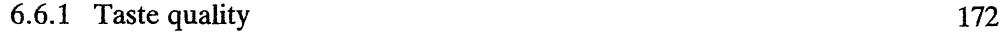

$\begin{array}{ll}\text { 6.6.2 Safety } & 174\end{array}$

$\begin{array}{ll}6.6 .3 \text { Solubility } & 175\end{array}$

$\begin{array}{ll}6.6 .4 \text { Stability } & 176\end{array}$

6.6.5 Cost 176

$\begin{array}{ll}6.7 \text { Conclusion } & 178\end{array}$

$\begin{array}{ll}\text { References } & 178\end{array}$

7 Sweetener markets, marketing and product development 186 M.G. LINDLEY

$\begin{array}{lll}7.1 & \text { Introduction } & 186\end{array}$

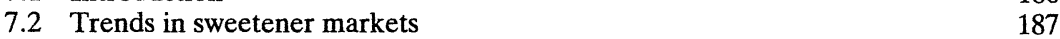

$\begin{array}{ll}7.2 .1 \text { Introduction } & 187\end{array}$

7.2.2 Nutritive sweetener markets 188

7.2.3 High intensity sweetener markets 190

$\begin{array}{ll}7.2 .4 \text { Conclusions } & 191\end{array}$

7.3 Sweetener marketing $\quad 191$

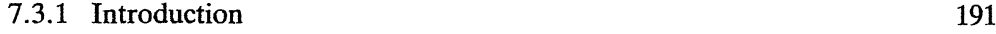

$\begin{array}{ll}\text { 7.3.2 Sweetener economics } & 192\end{array}$

$\begin{array}{ll}\text { 7.3.3 Nutritional aspects of sweetener marketing } & 193\end{array}$

$\begin{array}{ll}\text { 7.3.4 Conclusions } & 194\end{array}$

$\begin{array}{lll}7.4 & \text { Sweeteners in product development } & 194\end{array}$

$\begin{array}{ll}\text { 7.4.1 Introduction } & 194\end{array}$

$\begin{array}{ll}\text { 7.4.2 Sweetness intensity } & 195\end{array}$

$\begin{array}{ll}\text { 7.4.3 Sweetness stability } & 198\end{array}$

$\begin{array}{ll}7.4 .4 \text { Sweetener functionality } & 200\end{array}$

$\begin{array}{ll}7.5 \text { Conclusions } & 202\end{array}$

References 202

8 Sweeteners and dental health 205

K. WENNERHOLM, C.-G. EMILSON and D. BIRKHED

8.1 Dental caries: a multi-factorial disease 205

8.2 Evaluation of cariogenic potential 206

$\begin{array}{lll}8.3 \text { Sucrose } & 207\end{array}$

$\begin{array}{ll}\text { 8.3.1 Bacteriological studies } & 207\end{array}$

$\begin{array}{ll}\text { 8.3.2 Animal studies } & 208\end{array}$

$\begin{array}{ll}\text { 8.3.3 Clinical studies } & 208\end{array}$

8.4 Low- or non-cariogenic sugar substitutes 209

$\begin{array}{ll}8.5 \text { Glucose, fructose and invert sugar } & 209\end{array}$

$\begin{array}{ll}\text { 8.5.1 Bacteriological studies } & 209\end{array}$

$\begin{array}{ll}\text { 8.5.2 Animal studies } & 210\end{array}$

$\begin{array}{ll}\text { 8.5.3 Clinical studies } & 210\end{array}$

$\begin{array}{lll}8.6 & \text { Lactose } & 211\end{array}$

$\begin{array}{ll}8.7 \text { Palatinose (isomaltulose) } & 211\end{array}$

$\begin{array}{ll}\text { 8.7.1 Animal studies } & 211\end{array}$

8.7.2 Clinical studies $\quad 211$

$\begin{array}{lll}8.8 \text { Sorbose } & 211\end{array}$ 
8.8.1 Bacteriological studies $\quad 211$

8.8.2 Animal studies $\quad 212$

$\begin{array}{ll}\text { 8.8.3 Clinical studies } & 212\end{array}$

$\begin{array}{ll}8.9 \text { Xylitol } & 212\end{array}$

8.9.1 Bacteriological studies $\quad 212$

$\begin{array}{ll}\text { 8.9.2 Animal studies } & 212\end{array}$

$\begin{array}{ll}\text { 8.9.3 Clinical studies } & 212\end{array}$

8.10 Sorbitol $\quad 213$

8.10.1 Bacteriological studies $\quad 213$

$\begin{array}{ll}\text { 8.10.2 Animal studies } & 214\end{array}$

$\begin{array}{ll}\text { 8.10.3 Clinical studies } & 214\end{array}$

8.11 Mannitol $\quad 214$

$\begin{array}{ll}\text { 8.11.1 Bacteriological studies } & 214\end{array}$

$\begin{array}{ll}\text { 8.11.2 Animal studies } & 214\end{array}$

$\begin{array}{ll}8.12 \text { Lactitol } & 215\end{array}$

8.12.1 Bacteriological studies $\quad 215$

$\begin{array}{ll}\text { 8.12.2 Animal studies } & 215\end{array}$

$\begin{array}{ll}\text { 8.12.3 Clinical studies } & 215\end{array}$

8.13 Maltitol 215

8.13.1 Bacteriological studies $\quad 215$

8.13.2 Animal studies $\quad 216$

8.13.3 Clinical studies $\quad 216$

$\begin{array}{ll}8.14 \text { Palatinit } & 216\end{array}$

8.14.1 Bacteriological studies $\quad 216$

8.14.2 Animal studies $\quad 216$

$\begin{array}{ll}8.15 \text { Lycasin } & 216\end{array}$

$\begin{array}{ll}\text { 8.15.1 Bacteriological studies } & 217\end{array}$

$\begin{array}{ll}\text { 8.15.2 Animal studies } & 217\end{array}$

$\begin{array}{ll}\text { 8.15.3 Clinical studies } & 217\end{array}$

$\begin{array}{ll}8.16 \text { Starch hydrolysate } & 217\end{array}$

8.17 Non-caloric sweeteners $\quad 218$

8.18 Comparison of various groups of sweeteners $\quad 219$

$\begin{array}{ll}8.19 \text { Sweeteners and caries prevention } & 219\end{array}$

$\begin{array}{ll}\text { References } & 220\end{array}$

9 Sweeteners and metabolic disorders 225

N. FINER

9.1 Introduction $\quad 225$

9.2 Nutritive sweeteners 225

9.2.1 Normal metabolism of sugars and polyols 225

9.2.2 Nutritive sweetener metabolism in disease states $\quad 230$

$\begin{array}{lll}9.3 & \text { Non-nutritive sweeteners } & 238\end{array}$

9.3.1 Aspartame 238

9.3.2 Other non-nutritive sweeteners

9.4 Conclusions $\quad 243$

References $\quad 243$

10 Sweeteners and body weight 248

D.A. BOOTH

10.1 A behavioural issue $\quad 248$

10.1.1 The chemistry of sweeteners 248

10.1.2 The biochemistry of body weight $\quad 250$

10.1.3 The psychology of sweeteners and body weight $\quad 252$

10.2 Mechanisms of action of sweeteners on intake 255 
10.2.1 Intake of plain saccharin or sugars 255

10.2.2 Acquired dislikes and likings for sweetness $\quad 256$

10.2.3 Context-dependence of acquired sensory preferences 257

10.3 Theoretical implications for body weight control 257

10.3.1 How might sugar consumption cause obesity? 258

10.3.2 Could low-calorie sweeteners help to reduce weight? 259

10.3.3 Can we prevent obesity and still use sweeteners? 259

10.3.4 Implications for marketing of sweetened foods and beverages $\quad 260$

$\begin{array}{ll}10.4 \text { Conclusion } & 261\end{array}$

References $\quad 262$

11 Sweeteners: statutory aspects 265

D.J. SNODIN

$\begin{array}{ll}11.1 \text { Introduction } & 265\end{array}$

11.2 Sweeteners in commercial use and in development 266

11.3 Regulation and evaluation of sweeteners 266

$\begin{array}{ll}\text { 11.3.1 General } & 266\end{array}$

$\begin{array}{ll}\text { 11.3.2 US approach } & 276\end{array}$

11.3.3 European Economic Community approach $\quad 279$

11.3.4 Codex Alimentarius 283

$\begin{array}{ll}11.4 \text { Bulk sweeteners: special considerations } & 286\end{array}$

$\begin{array}{ll}11.5 \text { Sweetener intake assessments } & 287\end{array}$

$\begin{array}{ll}\text { 11.6 The ADI concept } & 289\end{array}$

$\begin{array}{ll}11.7 \text { Future trends } & 292 \\ & 293\end{array}$

References 293

$\begin{array}{lr}\text { Index } & 295\end{array}$ 